\title{
Feedback as a means to improve clinical competencies: Consultants' perceptions of the quality of feedback given to registrars
}

\author{
C I Bagwandeen, ${ }^{1} \mathrm{MB}$ ChB, DHSM, DOH, Dip HIV Man (SA), FCPHM; V S Singaram, ${ }^{2}$ BMedSc, MMedSc, PhD \\ ${ }^{1}$ Discipline of Public Health, School of Nursing and Public Health, College of Health Sciences, University of KwaZulu-Natal, Durban, South Africa \\ ${ }^{2}$ Clinical and Professional Practice, School of Clinical Medicine, College of Health Sciences, University of KwaZulu-Natal, Durban, South Africa
}

Corresponding author: C I Bagwandeen (bagwandeenc@ukzn.ac.za)

Background. Effective supervision by consultants in postgraduate medical education involves the process of feedback. Giving feedback may be challenging for consultants who have no formal training in this process, which may be further compounded in heterogeneous diverse settings.

Objective. To explore consultants' perceptions of feedback to registrars in a multicultural, multilingual diverse academic hospital setting.

Methods. Thirty-seven consultants consented to completing a questionnaire on what, when, where, how often, and how feedback was provided, as well as on the type and effect of feedback to registrars. Descriptive statistics were used to analyse the data. Differences between groups were calculated using Pearson's $\chi^{2}$ test for independent variables, with a $p$-value of $<0.05$ regarded as being statistically significant.

Results. Only $40 \%$ of consultants reported that they provided feedback often or always and $62.2 \%$ reported that standards were not predetermined and communicated to registrars. When feedback was provided, it was based on concrete observations of performance (78.4\%), it incorporated a plan for improvement (72.9\%) and it supplied information on techniques performed incorrectly (72.9\%). Only $40.5 \%$ of consultants provided feedback on procedures performed correctly. Moreover, only half of the consultants believed they were proficient at giving feedback.

Conclusion. Consultants need to develop the art of giving feedback through appropriate training so that they are more comfortable and proficient with the various aspects of feedback, leading to a positive effect on enhancing registrar training.

Afr J Health Professions Educ 2016;8(1 Suppl 1):113-116. DOI:10.7196/AJHPE.2016.v8i1.758

Effective supervision in postgraduate medical education involves the process of feedback, which may - in practice - be ineffective or inconsistent. ${ }^{[1]}$ Based on the literature, ${ }^{[2-6]}$ this study defines feedback as 'a process whereby the desired standard of proficiency in a task has been clearly established. This standard has been communicated to the student. Gaps in performing the task or level of knowledge are identified, and the student is made aware of his or her shortcomings, together with a plan to improve performance.' As medical education has moved beyond the paradigm of 'see one, do one, teach one, ${ }^{\text {[7] }}$ consultants need to be capable of providing suitable training guidance to ensure that graduates are clinically and otherwise competent. This should have a positive effect on patient outcomes, foster a life-long love of learning and the process of reflection, and promote good ethical practice. This process involves more than didactic input. It includes suitable feedback, so that competencies may be enhanced and improved and deficiencies corrected. ${ }^{[8]}$ It might be argued that if some (or all) of the elements contained in the definition are missing, feedback is not being adequately provided in clinical settings, thus affecting the calibre of specialists subsequently produced.

Feedback has been well recognised as an important component of education and can have an extremely powerful and positive effect on learning. ${ }^{[2,8,9]}$ It is regarded as integral and essential to postgraduate medical education, ${ }^{[4]} \mathrm{a}$ concept that is similar to that of serving an old-fashioned apprenticeship in an experiential learning setting. Without feedback, poor performance is not corrected, good performance is not entrenched and magnified, and no plans for improvement are implemented. ${ }^{[10,11]}$ Feedback that meets all the defined criteria can positively influence the performance of doctors. ${ }^{[12]}$ The importance of suitable external feedback by consultants to registrars becomes critical when there is no self-assessment by registrars or if the feedback is inaccurate. ${ }^{[13]}$ Giving feedback may be challenging for consultants who have no formal training in the process, which may be further compounded in heterogeneous settings involving students of different gender, ethnicity, race, socioeconomic backgrounds, educational levels and home or first languages. ${ }^{[14]}$ Consultants need to be sensitive to the different dynamics at play to ensure that the same message 'transmitted is received and understood $^{[15]}$ by the different groups in the same way.

Furthermore, several authors have reported that consultants often believe that they provide adequate, timeous and sufficient quality feedback, despite evidence from registrars indicating the contrary. ${ }^{[4,8,10,13]}$ Given the importance of feedback as an essential component of medical education, this discrepancy is of great concern and needs to be monitored. Hence, this study was undertaken to explore the consultants' and registrars' perceptions of feedback given and received. The study focuses on the perceptions of consultants with regard to the quality of feedback they provided to registrars employed at an academic hospital.

\section{Methods}

A questionnaire was designed to ascertain the consultants' perceptions on what, when, where, how often, and how feedback was provided, as well as on the type and effect of feedback to registrars. Sociodemographic information (age, gender, home language, discipline and years of specialisation) was also gathered. A definition of feedback, as discussed above, was also included in the questionnaire to try to prevent any misconceptions with regard to the basic tenets of this process. Responses were reported on a 5-point Likert scale (Figs 1 and 2). Although this observational study adopted a mixed-methods approach, this article focuses on the quantitative data used to survey the overall perceptions of the consultants. The qualitative investigation of these perceptions will be reported in a follow-up study. 


\section{Research}

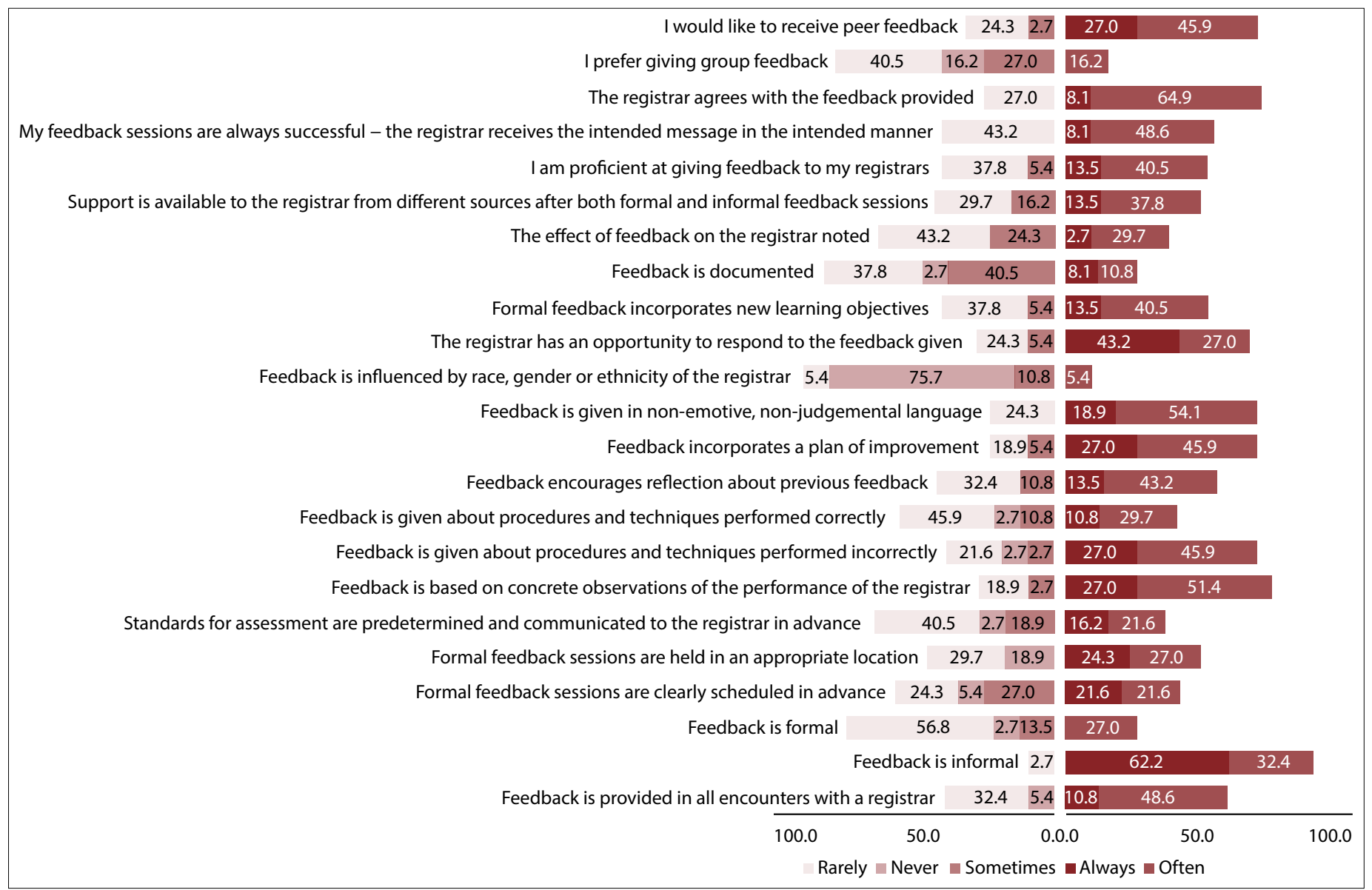

Fig. 1. A divergent stacked bar graph showing consultants' perceptions on the feedback they give to registrars.

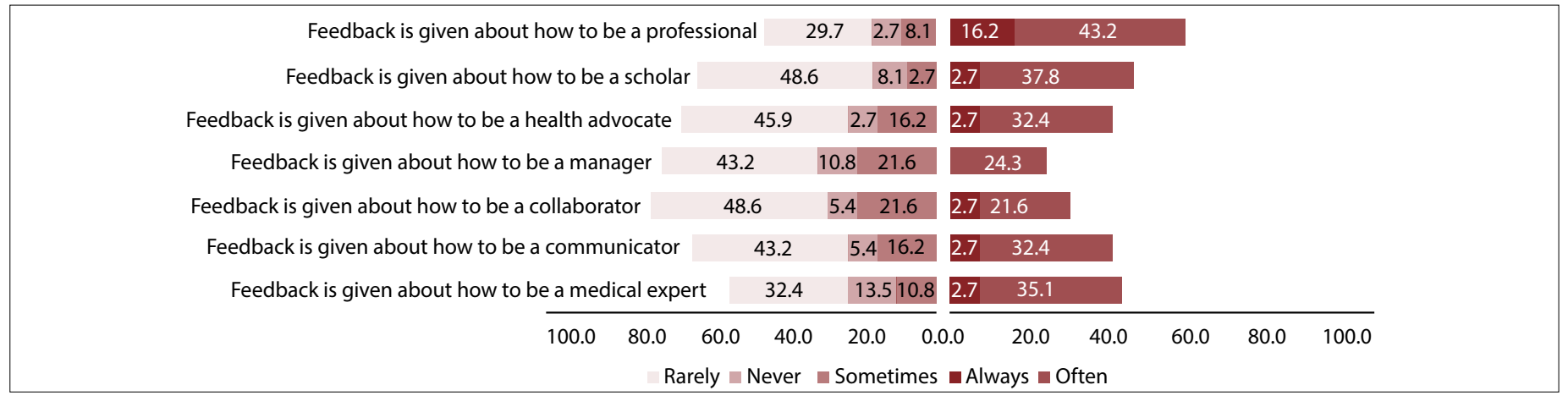

Fig. 2. A divergent stacked bar graph showing consultants' perceptions on the feedback they give to registrars with regard to graduate attributes.

All consultants from the disciplines of Surgery, Internal Medicine, Obstetrics and Gynaecology, Paediatrics, Psychiatry and Family Medicine were invited to participate. Because of a $0 \%$ response rate to the online questionnaire, hard copies were distributed at academic day meetings; $62 \%(n=37)$ were returned anonymously with informed consent. Descriptive statistics were used to interpret the responses of the registrars, with mean values calculated. Differences between groups were calculated using Pearson's $\chi^{2}$ test for independent variables, with a $p$-value of $<0.05$ regarded as sta- tistically significant. Ethical approval for the study was granted by the Humanities and Social Sciences Ethical Committee, University of KwaZulu-Natal, Durban, South Africa (HSS/1185/013D).

\section{Results}

The mean age of the consultants was 37.8 (range 31 55) years. Fifty-four percent were female. English was the first language for the majority of consultants $(n=31)$, while six spoke another language. Six consultants had qualifications other than the Colleges of Medicine of South Africa Fellowship in their respective fields: 1 had a $\mathrm{PhD}$ in Surgery, while the other 5 had postgraduate certificates in their respective fields. Thirteen consultants from Paediatrics, 9 from Internal Medicine, 8 from Obstetrics and Gynaecology, 3 from Surgery and 2 each from Psychiatry and Family Medicine responded.

As illustrated in Fig. 1, 62.2\% of consultants reported that standards for assessment were not predetermined and communicated to registrars in advance - always or often. All consultants reported that they provided feedback, but only 
$\sim 40 \%$ provided feedback always or often. The majority of consultants based their feedback on concrete observations of registrar performance (78.4\%), incorporated a plan for improvement in their feedback $(72.9 \%)$, or provided feedback on techniques performed incorrectly (72.9\%). However, only $40.5 \%$ provided feedback on procedures performed correctly, while $56.7 \%$ thought that feedback encouraged reflection about previous feedback. The vast majority gave informal feedback (94.6\%). Only $27 \%$ gave formal feedback that was clearly scheduled in advance, given in an appropriate location and that incorporated new learning objectives - only half of the time. Feedback given was influenced by race, gender or ethnicity of the registrars - sometimes (10.8\%) and often (5.4\%). Seventy-three percent reported that feedback was given using non-emotive, non-judgemental language, and $70.2 \%$ of consultants gave registrars an opportunity to respond to feedback, but only $32.4 \%$ noted the effect of feedback on the registrar. A total of 51.3\% reported that support for registrars was available after feedback. While $73 \%$ of the consultants felt that the registrar agreed with the feedback, 54\% reported that they were proficient at giving feedback and believed that their intended message was received. Most consultants $(83.7 \%)$ preferred giving feedback one on one, and would have liked to receive peer feedback $(72.9 \%)$.

Registrars need feedback on both technical and other specific skills and on graduate attributes to improve outcomes. With regard to technical skills, feedback on how to be a professional was provided always or often (59.4\%), while the specifics around being a medical expert (56.7\%), communicator $(64.8 \%)$, collaborator $(75.6 \%)$, manager $(75.6 \%)$, health advocate $(64.8 \%)$, and scholar $(59.4 \%)$ were neglected. Sixty-two percent of consultants believed that they always or often provided feedback about clinical skills, technical skills and evidence-based practice, but feedback about interpersonal skills (67.6\%), communication skills (59.5\%) and ethics (54\%) was rarely or never provided.

This study found that consultants delivered feedback in a variety of settings. While no consultants provided feedback during group teaching, $84 \%$ gave feedback during academic days, $62 \%$ made use of side-room settings, $50 \%$ provided one-on-one feedback, and $32 \%$ gave feedback at the bedside. No statistically significant relationship was observed between the age of consultants and how they perceived feedback to be provided. With regard to gender, male consultants believed that they were proficient at giving feedback, significantly more than their female counterparts $(p=0.041$, mean 21.91).

Consultants whose mother tongue was English showed significant differences compared with other language speakers, as they gave more feedback about how to be a communicator ( $p=0.031$, mean 20.58), a collaborator $(p=0.017$, mean 20.74) and a manager ( $p=0.052$, mean 20.44). Provision of feedback was significantly influenced by race, gender and ethnicity of registrars, more so in consultants who were English secondlanguage speakers ( $p=0.05$, mean 27.58) than English first-language speakers.

\section{Discussion}

The importance of providing feedback in registrar training has been well documented. ${ }^{[1-5]}$ A good approach to feedback is essential and several necessary elements have been identified for successful feedback to occur and ensure that the process attains the desired end result of improving performance. ${ }^{[2,4,16,17]}$ Two of the fundamental requirements for an appropriate and adequate feedback process involve: (i) the development of the desired stan- dards to be obtained, and for these standards to be clearly communicated to the registrar in advance; and (ii) that the consultant's feedback be based on direct observation of the registrar's performance and compared with the desired standard to be achieved. Such feedback must include an improvement plan to overcome any deficiencies between actual and desired performance. ${ }^{[3,4]}$ The majority of consultants did not communicate such desired 'gold standards' to the registrars. Furthermore, more than one-quarter did not base their observations on direct observation of performance or provide a plan for improvement in the feedback given. Therefore, registrars did not always have a clearly defined set of rules as a benchmark. These findings highlight essential fundamental flaws in the current practice of feedback across the disciplines at our academic hospital. This study suggests that each department should develop a set of guidelines that should be given to registrars at the beginning of a rotation, and consultants should be made aware that the feedback process hinges on direct observation of performance and incorporates improvement plans. Moreover, while feedback is used to correct deficiencies, it should also enhance good performance. The majority of consultants did not give feedback on procedures performed correctly, hence missing the opportunity to cement good practice. ${ }^{[5]}$

All consultants reported that they provided feedback, but the majority provided it infrequently and informally. With this approach, registrars will not always recognise feedback as feedback, and may not pay as much attention to it as when it is formally scheduled in advance. ${ }^{[1]}$ Owing to the experiential nature of the clinical teaching setting, it is of concern that consultants do not optimise all opportunities with the registrar to provide feedback. This is an indication that many teaching opportunities are being lost. Many consultants provided teaching at the bedside, a valuable setting for practical demonstration of clinical skills. However, academic days, which could be the best time for emphasising 'softer' skills, including graduate attributes, professionalism and ethics, were not maximised. A specific time should be set aside for discussions around such aspects on these days. Also, care should be taken to highlight the relevant application of such tenets during case presentations or didactic lectures on the effect that key areas have on clinical care to ensure that graduates are equipped with more than clinical competencies. ${ }^{[17]}$

As feedback has been likened to giving bad news, the effect of the message on the recipient cannot be ignored. ${ }^{[5]}$ This is particularly important in the diverse multicultural setting of this study. Of note, the majority of consultants were not influenced by the race, gender or ethnicity of the registrar. However, this issue needs to be addressed, as not all consultants reported that feedback was given in a non-emotive and non-judgemental way. This is a major concern and counteracts the purpose of giving feedback - to improve performance - as registrars should not be in a position of reacting to how something was being said, rather than what was being said, and so losing the intended message. ${ }^{[5]}$

Similarly, not noting the effect of consultants' feedback on registrars, could have a harmful result. In the face of negative criticism, some registrars lack the emotional capacity to recover from this and may flounder in their attempts to improve on their performance. ${ }^{[5,18]}$ Conversely, others, particularly those with strong personalities, may choose to believe that their consultants are incorrect and persist in their chosen behaviour. ${ }^{[18}$ It is therefore vital not only to be cognizant of the effect of both formal and informal feedback, but also to ensure referral to appropriate support structures should these be required; however, only $45.9 \%$ of consultants were aware of the support structures that registrars could access or be 
referred to. While the majority of consultants gave registrars an opportunity to respond to feedback, when this did not occur misunderstandings and misconceptions were not clarified.

Consultants and students need to be skilled in the art of giving feedback. ${ }^{[2,46]}$ Only half of the consultants felt that they were proficient in providing feedback and gave feedback often. Also, less than a third gave feedback about technical skills. As providing feedback is key to improving academic outcomes and clinical proficiency, inadequacies in being able to provide feedback generally and about essential competencies highlight a gap in the key performance areas of consultants and indicate the need for staff development, in addition to a possible postgraduate clinical qualification for employment in an academic teaching hospital.

It is encouraging that all consultants agreed that feedback was essential to registrar training and the vast majority felt that they should be trained to give feedback. Consultants are aware of the importance of feedback in honing relevant skills and of their own deficiencies and the need to rectify these through appropriate training. The race, gender and ethnicity of the registrars affected the provision of feedback significantly more for English second-language consultants than for English first-language speakers. The latter consultants were probably more aware of the barriers that non-proficiency in the medium of instruction could pose and took care to overcome them. Conversely, given that all communication between registrar and consultant is in English, the consultants for whom English was their home language believed that they were skilled in providing feedback, possibly because of their ease of use of the language. Consequently, they did not pay as much attention to ensuring that feedback was as successful as it should be, especially for registrars who were not as proficient in English as they were. However, regardless of race or language, generally male consultants believed that they were more proficient at providing feedback than female consultants.

\section{Conclusion}

The study found that the art of giving and receiving feedback has to be nurtured so that consultants are more comfortable with and proficient in the process, not only in specific skills, but also with regard to essential graduate outcomes. To train consultants in this process would entail a form of continuing professional development, especially as they are recruited on their clinical skills and the assumption that knowing how to do a procedure equates to being able to communicate it well, without any formal exposure to didactic instruction. This would encourage a process of reflection and seeking feedback from registrars, starting in the preclinical years. An integral component of this training would have to be recognising the effect of feedback on registrars, so that any undesirable outcomes could be appropriately dealt with, be it refusal to accept the feedback or negative emotional reactions. Support structures and mechanisms must be developed internally by disciplines and the university at large, and referral pathways must be developed and communicated to consultants and registrars so that they are able to access these quickly and confidentially if and when required. While it is gratifying that most consultants were able to embrace the multicultural and diverse setting, a small majority appeared to be affected by race, gender and ethnicity. We recommend that appropriate programmes addressing diversity issues be implemented so that no-one is prejudiced by these apparent biases.

Acknowledgements. This article was made possible by grant No. R24TW008863 from the Office of the US Global AIDS Coordinator and the US Department of Health and Human Services, National Institutes of Health (NIH OAR and NIH ORWH). Its contents are solely the responsibility of the authors and do not necessarily represent the official views of the US government. We wish to thank Dr M Muzigaba for help with statistical analysis, and the staff and students who participated in this study.

\section{References}

1. Busari JO, Weggelaar N, Knottnerus AC, Greidanus P, Scherpbier AJIA. How medical residents perceive the quality of supervision provided by attending doctors in the clinical setting. Med Educ 2005;39:696-703. quality of supervision provided by attending doctors in the clinical setting. Med
Hattie I, Timperley H. The power of feedback. Rev Educ Res 2007;77:81-112.

2. Hattie J, Timperley H. The power of feedback. Rev Educ Res 2007;77:81-112.
3. Van de Ridder JM, Stokking K, McGaghie W, ten Cate O. What is feedback in clinical education? Med Educ Van de Ridder JM, Stokking K, McGaghie W, ten Cate O. What is fe
2008;42:189-197. [http://dx.doi.org/10.1111/j.1365-2923.2007.02973.x]

4. Ende J. Feedback in medical education. JAMA 1983;250:777-781.

5. DeLima TJ, Arnold R. Giving feedback. J Palliat Med 2011;14(2):233-239. [http://dx.doi.org/10.1089/ jpm.2010.0093]

6. Shrivastava SR, Shrivastava PS, Ramasamy J. Effective feedback: An indispensable tool for improvement in quality of medical education. J Pedagog Dev 2014;4(1):12-20.

7. Rodriguez-Paz JM, Kennedy E, Salas E, et al. Beyond 'see one, do one, teach one': Toward a different training paradigm. Qual Saf Health Care 2009;18:63-68. [http://dx.doi.org/10.1136/qshc.2007.023903]

8. Menachery EP, Knight AM, Kolodner K, Wright SM. Physician characteristics associated with proficiency in feedback skills. J Gen Intern Med 2006;21:440-446.

9. Norcini I. The power of feedback. Med Educ 2010;44:16-17. [http://dx.doi.org/10.1111/j.1365-2923.2009.03542.x]

10. Lieberman A, Lieberman M, Steinert Y, McLeod P, Meterissian S. Surgery residents and attending surgeons have different perceptions of feedback. Med Teach 2005;27:470-472.

11. Cantillion P. Giving feedback in clinical settings. BMJ 2008;337:a1961. [http://dx.doi.org/10.1136/bmj.a1961]

12. Veloski J, Boex J, Grasberger M, Evans A, Wolfson D. Systematic review of the literature on assessment, feedback and physicians' clinical performance: BEME Guide No. 7. Med Teach 2006;28:117-128.

13. Kogan J, Conforti LN, Bernabeo EC, Durning SJ, Hauer KE, Holmboe ES. Faculty staff perceptions of feedback to residents after direct observation of clinical skills. Med Educ 2012;46:201-245. [http://dx.doi.org/10.1111/j.13652923.2011.04137.x]

14. Holen A. The PBL group: Self-reflections and feedback for improved learning and growth. Med Teach 2000;22:485-488.

15. Murdoch-Eaton D. Feedback: The complexity of self-perception and the transition from 'transmit' to 'received and understood'. Med Educ 2012;46:538-540. [http://dx.doi.org/10.1111/j.1365-2923.2012.04278.x]

16. Bing-You R, Trowbridge R. Why medical educators may be failing at feedback. JAMA 2009;32:1330-1331. [http:// dx.doi.org/10.1001/jama.2009.1393]

17. Fluit $\mathrm{C}$, Bolhuis $\mathrm{S}$, Grol R, et al. Evaluation and feedback for effective clinical teaching in postgraduate medical education: Validation of an assessment instrument incorporating the CanMEDS roles. Med Teach 2012;34:893-101. 8. Archer J. State of the science in health professional education: Effective feedback. Med Educ 2010;44:101-108. [http://dx.doi.org/10.1111/j.1365-2923.2009.03546] 